\title{
COMPARISON OF TRANSONIC FLOWS THROUGH TWO TIP-SECTION TURBINE BLADE CASCADES WITH DIFFERENT FLAT PROFILES
}

\author{
Josef Musil ${ }^{1,2}$, Jaromír Příhoda ${ }^{1}$, Jiří Fürst ${ }^{2}$, Martin Luxa ${ }^{1}$, David Šimurda ${ }^{1}$ \\ ${ }^{1}$ Institute of Thermomechanics, Czech Academy of Sciences, Dolejškova 5, 18200 \\ Praha 8, Czech Republic \\ ${ }^{2}$ Faculty of Mechanical Engineering, Czech Technical University in Prague, Karlovo \\ nám. 13, 12135 Praha, Czech Republic
}

\begin{abstract}
The contribution deals with numerical simulations of 2D compressible flow through two variants of the tip-section turbine blade cascade with a flat profile for the subsonic inlet. Simulations were realised by means of the OpenFOAM code using the $\gamma-R e_{\theta}$ bypass transition model completed by the two-equation SST turbulence model. Predictions were focused particularly on the impact of the shock-wave/boundary-layer interaction on the flow structure in the inter-blade channel. Beside the effect of the inlet angle on the flow around the leading edge was studied. Numerical results were compared with optical and pressure experimental data.
\end{abstract}

Keywords: supersonic tip-section blade cascade, CFD, transition modelling

\section{Introduction}

The last stage of the low-pressure part of the steam turbine of the large output is usually formed by very long blades. Because of the high circumferential velocity the flow through the tip-section blade cascade is mainly supersonic. Recent results have demonstrated the influence of the shock-wave interaction with the boundary layer on the flow structure in the blade cascade and energy losses as well, see Luxa et al. [1]. Existing research was focused mainly on the shock-wave/boundary-layer interaction with the turbulent boundary layer. An overview of experimental and numerical investigation of the shock-wave/boundarylayer interaction is given by Gaitonde [2]. Results related to the experimental investigation of the interaction of the shock wave with the laminar boundary layer were published e.g. by Giepman et al. [3]. Besides experimental studies, there are also some attempts to simulate separation-induced transition due to the shock wave/boundary-layer interaction by means of the RANS and LES approaches as well, see e.g. Teramoto [4], Piotrowicz and Flaszyński [5].

The contribution deals with the numerical simulation of 2D transonic flows through two tip-section turbine blade cascades with subsonic inlet boundary conditions and it is a follow up to simulations made by Luxa et al. [6] and Straka et al. [7]. The both blade cascades represent variants of the rotor turbine blade tip-section with different flat profiles and were experimentally investigated in a wide range of Mach numbers $M_{1}$ and $M_{2 i s}$, see Luxa et al. [8, 9]. The cascade TR-U-9 was proposed for design conditions with the subsonic inlet and so the comparison of both cascades was realized for the inlet Mach number $M_{1}=0.8$ and the isentropic outlet Mach number $M_{2 i s}=1.8$ corresponding to the pressure in the settling chamber. The blade profile is sensitive to the inlet flow angle, especially at the supersonic inlet where the connection of the inlet flow angle and the inlet Mach number exists, see Musil et al. [10]. The influence of the various inlet flow angle on the flow near the leading edge of both blade profiles was tested at the subsonic inlet as well. Simulations were carried out by the OpenFOAM code using the Favre-averaged Navier-Stokes equations completed by the two-equation SST turbulence model and the $\gamma-\operatorname{Re}_{\theta}$ bypass transition model.

\section{Mathematical model}

Simulations of compressible flows were accomplished by the conditionally-averaged Navier-Stokes equations completed by constitutional relations for the ideal gas, the two-equation SST turbulence model of Menter [11] and by the bypass-transition $\gamma-R e_{\theta t}$ model of Langtry and Menter [12]. The constant turbulent Prandtl number $\operatorname{Pr}_{t}=$ const. is used in the simplest turbulent heat transfer model. 
The transition model is based on transport equations for the intermittency coefficient and the local transition momentum Reynolds number. The intermittency coefficient is given by the equation

$$
\frac{\partial(\rho \gamma)}{\partial t}+\frac{\partial\left(\rho U_{j} \gamma\right)}{\partial x_{j}}=P_{\gamma}-E_{\gamma}+\frac{\partial}{\partial x_{j}}\left[\left(\mu+\frac{\mu_{t}}{\sigma_{\gamma}}\right) \frac{\partial \gamma}{\partial x_{j}}\right]
$$

The production term

$$
P_{\gamma}=F_{\text {length }} \mathrm{C}_{a 1} \rho S\left(\gamma F_{\text {onset }}\right)^{1 / 2}\left(1-\mathrm{C}_{e 1} \gamma\right)
$$

contains empirical correlations $F_{\text {onset }}$ for the transition onset and $F_{\text {length }}$ for the transition length. The destruction term $E_{\gamma}$ allows the prediction of the re-laminarization of the boundary layer during a strong acceleration of the flow. The empirical correlation for the transition onset $F_{\text {onset }}$ depends on the critical Reynolds number $R e_{\theta c}$. The parameters $R e_{\theta c}$ and $L_{\text {length }}$ are expressed by means of the local Reynolds number $\overline{R e} \theta t$ given by the transport equation. The application of local variables is realized by the relation between the Reynolds number related to the momentum thickness and the maximum of the vorticity Reynolds number. The model is switched over to a simple algebraic transition model in the case of the transition in separated flow. Due to local variables the transition model can be used for the simulation of complex flows using unstructured computational grids.

Simulations were performed by using open-source software package OpenFOAM with the in-house LU-SGS solver for turbulent compressible flows developed by Fürst [13] with the first order implicit scheme in time, the second order upwind scheme for convection and the second order linear scheme for viscous fluxes.

The scheme of the computational domain taking one pitch of the blade cascade is shown in Figure 1. Inlet boundary conditions are prescribed by the constant total pressure, total temperature and inlet flow angle. The outlet boundary condition is defined by the constant static pressure determined according to the outlet isentropic Mach number. Periodicity conditions were used on side boundaries of the computation domain. The inlet free-stream turbulence parameters are given by the turbulence intensity $T u=1.5 \%$ and the ratio of turbulent and molecular viscosity $\mu_{\ell} / \mu=100$.

The computational grid contains about 150000 cells of the rectangular shape in boundary layer and of quadrilateral and triangular shapes in free stream. The nearest node is in the distance from the wall about $n^{+} \approx 1$. The detail of the mesh around the leading edge is given in Figure 2.

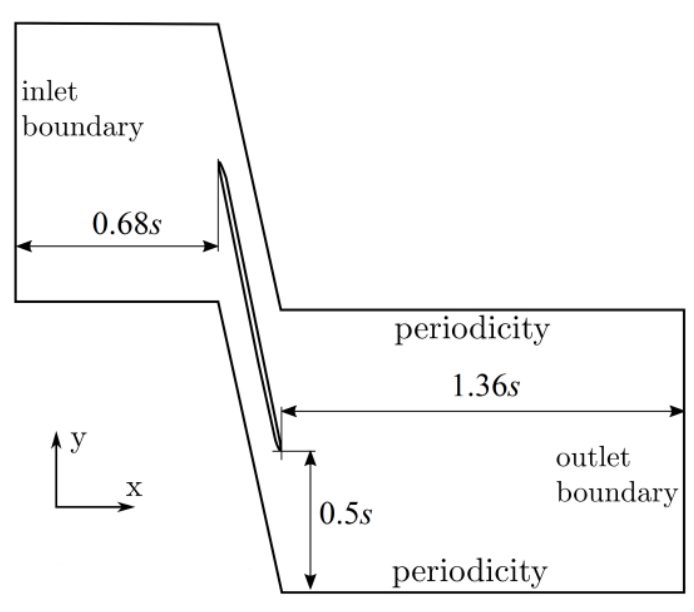

Figure 1: Scheme of the computational domain

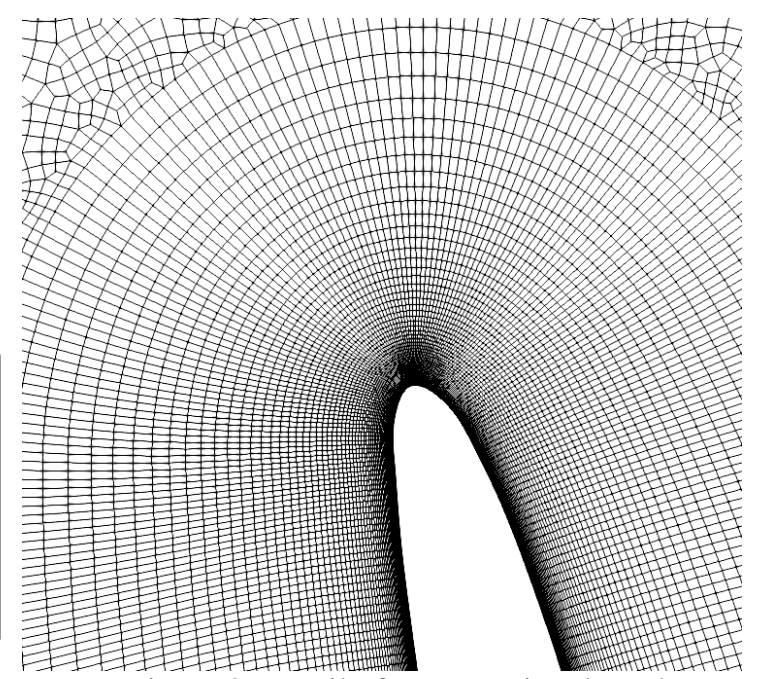

Figure 2: Detail of computational mesh

\section{Results}

Numerical simulations of 2D transonic flows were realized for the TR-U-8 and TR-U-9 blade cascades. The profiles of blades are designed flat with differently shaped leading and trailing parts. Both blades have the same profile chord $c=0.15 \mathrm{~m}$, the relative pitch $s / c=0.904$ and the maximum relative thickness 
$t / c \approx 0.02$. The stagger angle is $\gamma=81.59^{\circ}$ for the TR-U-8 cascade and $\gamma=79.23^{\circ}$ for the

TR-U-9 cascade. The stagger angle was measured from the blade suction side. Simulations were carried out for the inlet Mach number $M_{1} \approx 0.8$ and the isentropic outlet Mach number $M_{2 i s} \approx 1.86$ corresponding to the $M_{2 i s S C}=1.8$ in the settling chamber behind the test section. The corresponding isentropic outlet Reynolds number was $R e_{2 i s} \approx 1.9 \times 10^{6}$. Similarly, as for other tip-section blade cascades pressure and suction sides are on the inverse blade sides than usually. The flow fields in the both blade cascades were compared at the same incidence angle $\imath=0.6^{\circ}$.

The interferometric and schlieren pictures of the flow field in the TR-U-8 blade cascade are shown in Figure 3. The corresponding computed field of Mach number isolines is given in Figure 4. The effect of the interaction of the inner branch of the exit shock wave of the neighbouring blade with the boundary layer on the suction side of the blade is well apparent in the interferometric pictures and in the computed field of the Mach isolines as well.

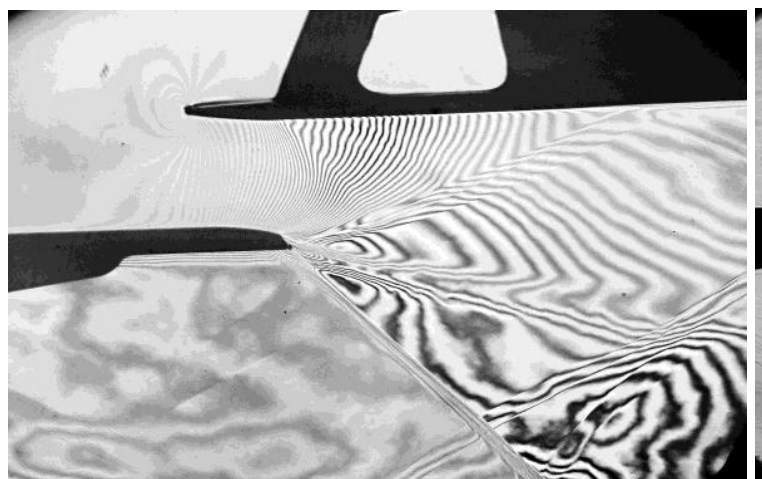

a) Interferometric picture

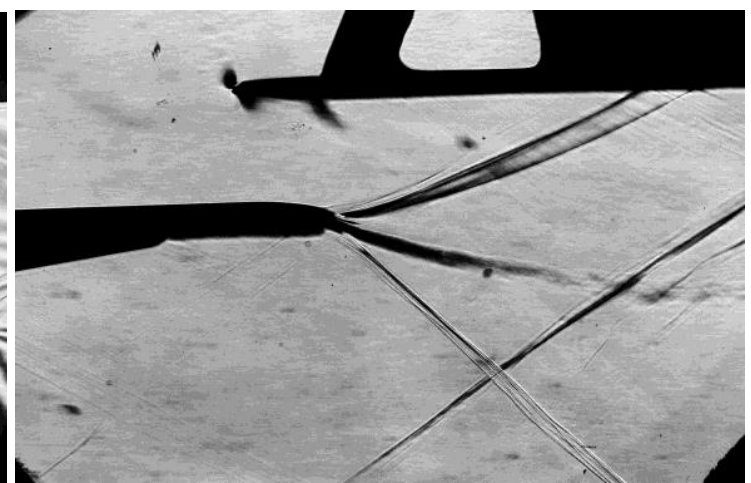

b) Schlieren picture

Figure 3: Flow field in the TR-U-8 blade cascade

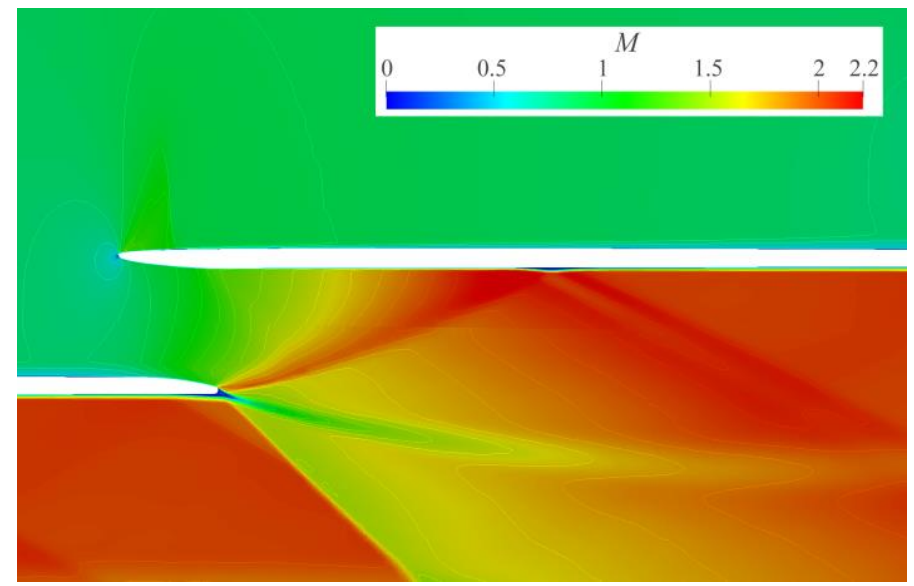

Figure 4: Mach number isolines in the TR-U-8 blade cascade

The interaction of the shock wave with the boundary layer takes place at the distance $x / b \approx 0.49$ according to the interferometric picture while at $x / b \approx 0.42$ in the simulation. The distance $b$ represents the axial chord $b / c=0.162$. It leads to the shift of the isentropic Mach number maximum on the blade suction side as can be seen on the distribution of the isentropic Mach number on the blade in Figure 5. As well the increase of the isentropic Mach number $M_{i s}$ to the trailing edge can be seen on the pressure side. Besides, the flow in the inter-blade channel is influenced by the sudden change of the surface curvature. The connection of the curved inlet part with the following straight part can be seen in Figure 4 .

The difference between the measured and computed inclination of the inner branch of the exit shock wave is given by the application of a simple computational domain with periodic boundary conditions on side walls. The better agreement can be achieved by using the computational domain according to the test section of the wind tunnel including the settling chamber, see Louda et al. [14]. 
Similarly, the interferometric and schlieren pictures from optical measurements of the TRU-9 blade cascade are given in Figure 6. As can be seen, the flow structure is in the both cases practically the same. The dominant effect on the flow structure has again the interaction of the exit shock wave with the boundary layer on the blade suction side. It follows from the interferometric and hot-film measurements (see Luxa et al. [9]) that the transition on the suction side could occur in the attached flow upstream the interaction of the boundary layer with the shock wave.

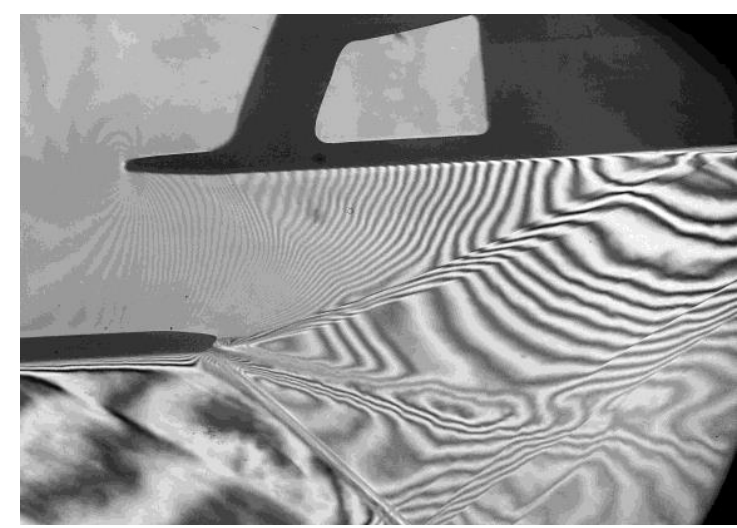

a) Interferometric picture

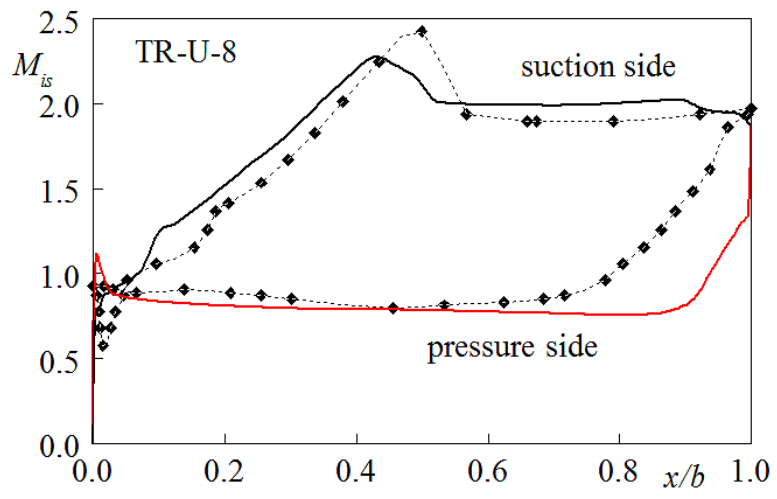

Figure 5: Distribution of the isentropic Mach number

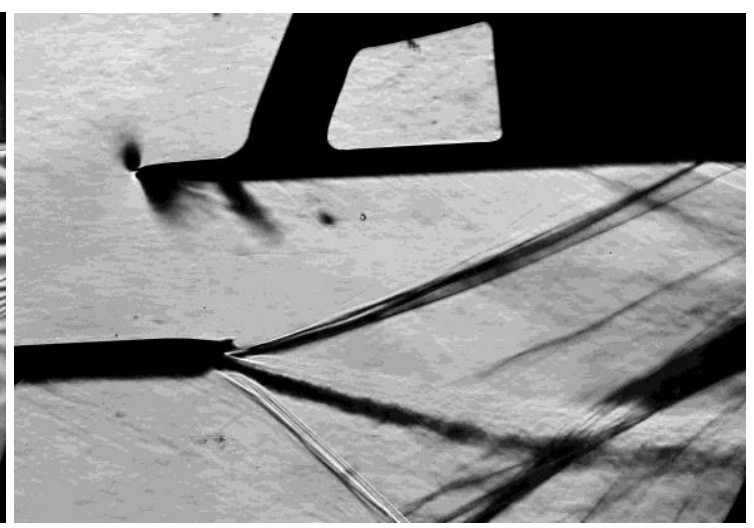

b) Schlieren picture

Figure 6: Flow field in the TR-U-9 blade cascade

The field of Mach number isolines computed for the TR-U-9 blade cascade is shown in Figure 7. The flow structure is very similar to the flow in the TR-U-8 blade cascade. The distribution of the isentropic Mach number in the TR-U-9 blade cascade blade is compared with measurement in the Figure 8.

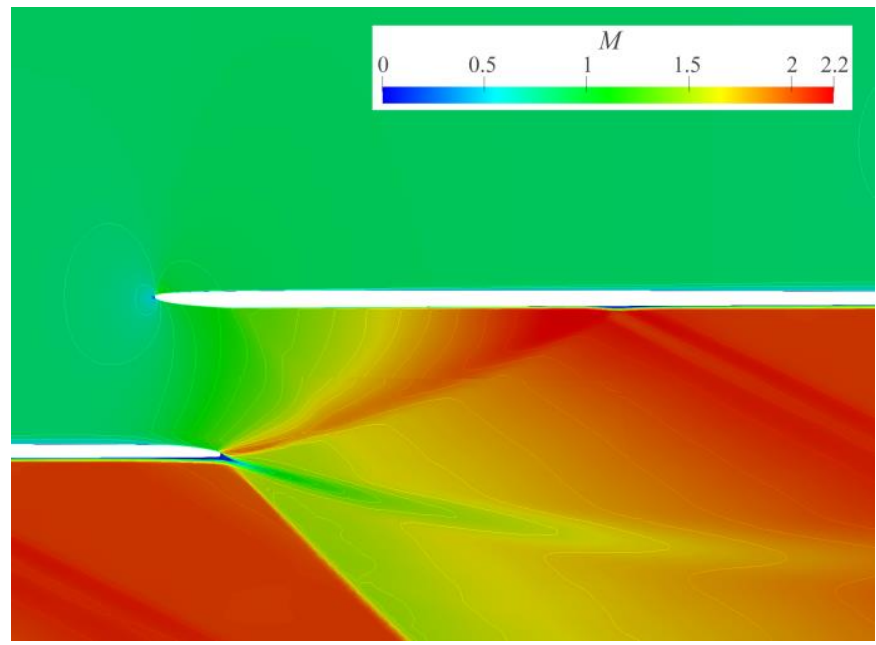

Figure 7: Mach number isolines in the TR-U-9 blade cascade

The reflections of shock waves from the side of the test section can be seen in interferometric and schlieren pictures for the both blade cascades. Therefore, the perforated tailboard placed behind the trailing edge of the side blade is used in some measurements. Due to the perforated tailboard the reflected shock waves are reduced so the energy losses determined from measurements behind the cascade are more believable. On the other side, the application of the tailboard leads to the higher outlet isentropic Mach number $M_{2 i s}$ and to the different angle of the exit shock wave. Therefore, the measurements without 
the tailboard are used for comparison.

The distribution of the isentropic Mach number $M_{i s}$ in the TR-U-9 blade cascade blade is shown in Figure 8. The course of $M_{i s}$ is again very similar as for the TR-U-8 cascade. A velocity peak on the pressure side behind the leading edge due to the positive incidence angle is followed by a practically constant Mach number with an increase upstream the trailing edge. The effect of the sudden change of the blade surface curvature and the interaction of the shock wave can be seen on the suction side. The difference between experimental data and the simulation in the position of the

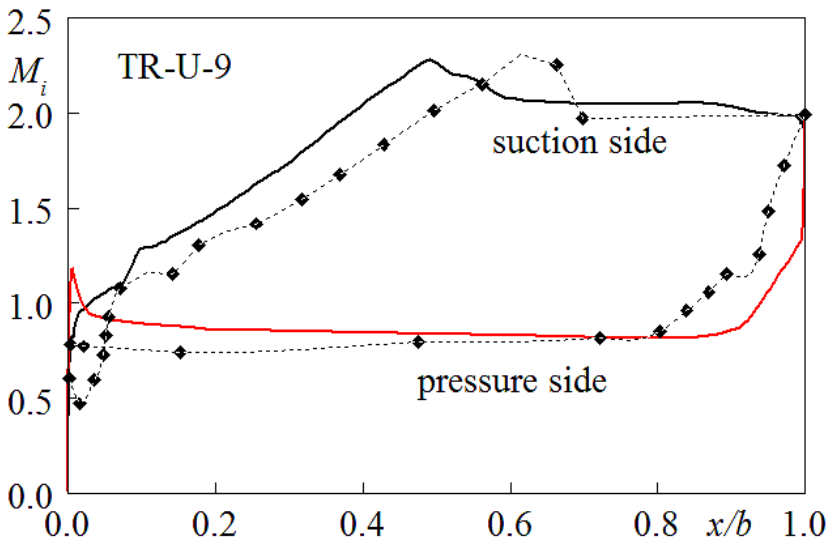

Figure 8: Distribution of the isentropic Mach number shock wave impact on the suction side is similar as at the TR-U-8 blade cascade.

The changes in the distribution of the isentropic Mach number correspond to the distribution of the skin friction coefficient given for the TR-U-9 cascade in Figure 9. The skin friction coefficient $C_{f}=\tau_{w} / p_{d l}$ is defined by the ratio of the wall friction and the inlet dynamic pressure. The short increase of $C_{f}$ on the suction side at the distance $x / b \approx 0.1$ is in accordance with the sudden change of the surface curvature. The interaction of the shock wave with the boundary layer leads at $x / b \approx 0.5$ to the flow separation followed by the transition in separated flow. The separation near the trailing edge is given by the curvature of the one side of the blade and it does not depend on the inlet flow angle. A short laminar separation bubble induced by the velocity peak can be seen near the leading edge on the pressure side. The transition to turbulence comes about downstream at the distance $\mathrm{x} / \mathrm{b} \approx 0.15$.

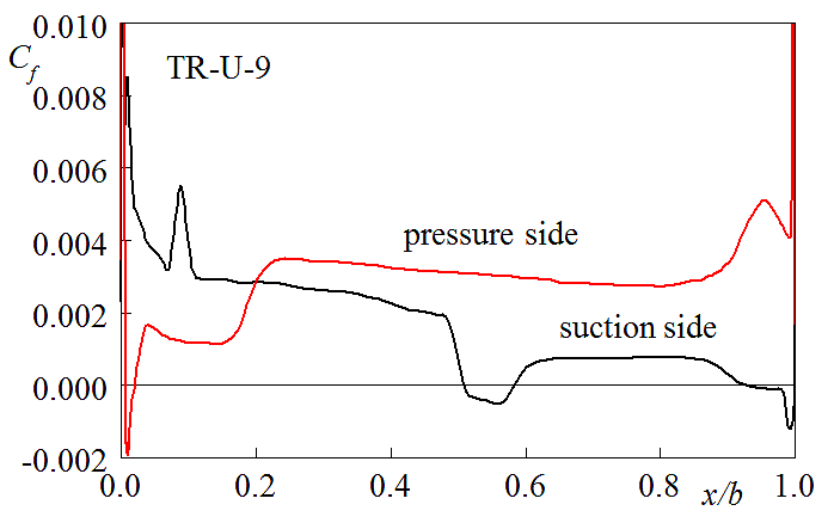

Figure 9: Skin friction distribution

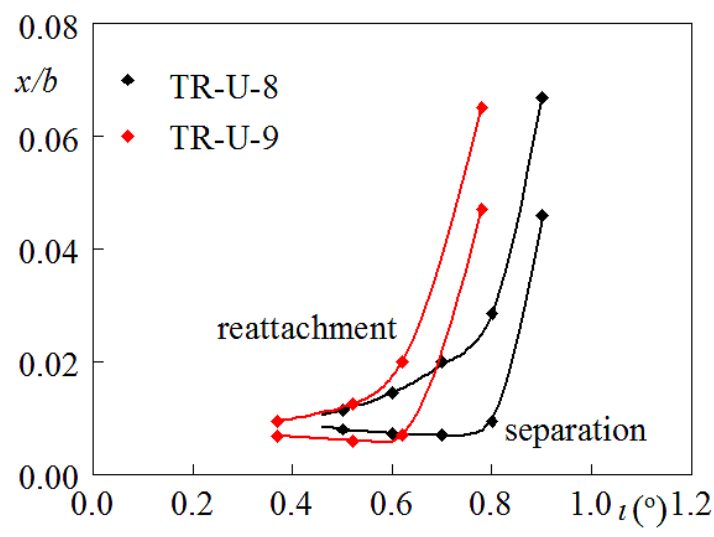

Figure 10: Separation bubble near the leading edge side
The variation of the skin friction with the incidence angle was studied on pressure side of both cascades. The velocity peak near the leading edge leads to a short laminar separation bubble followed downstream by the transition in attached flow. The extent of the separation is shown in Figure 10. Due to different curvature of the leading edge the separation bubble is shifted to the higher incidence angle for the TR-U-8 cascade. The separation bubble moves downstream with the increasing incidence. The transition onset in attached flow is shown in Figure 11. This transition moves on the contrary upstream for the increasing incidence angle.

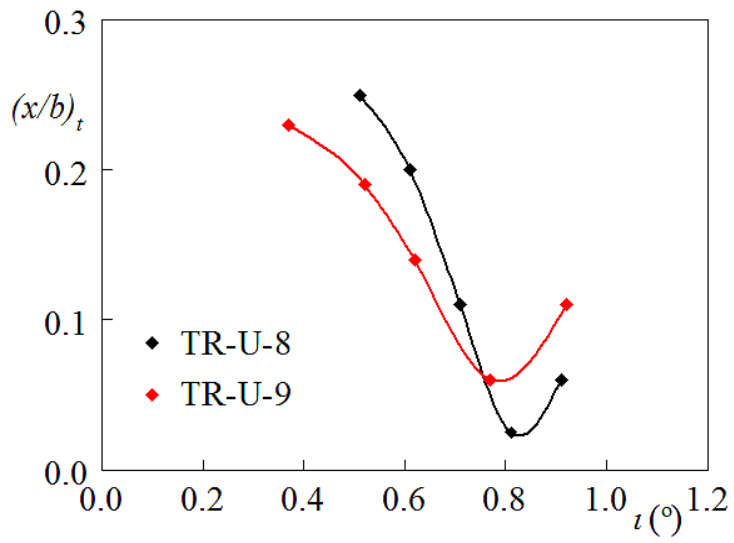

Figure 11: Transition onset on the pressure 
Unlike the separation near the leading edge the inlet angle does not influence the position and the extent of the separation zone due to the interaction of the shock wave with the boundary layer on the suction side.

\section{Conclusions}

Numerical simulations of 2D transonic flow through two variants of the tip-section turbine blade cascade with a flat profile were completed for the subsonic inlet. The two-equation SST turbulence model with the $\gamma-R e_{\theta}$ bypass transition model implemented in the OpenFOAM code was used for the comparison of flows through cascades with profiles differing only in curvatures of leading and trailing edges. Predictions were concentrated partly on the impact of the shock-wave/boundary-layer interaction on the flow structure in the inter-blade channel and partly on the effect of the inlet angle on the flow around the leading edge. The interaction of the inner branch of the exit shock-wave with the boundary layer connected with flow separation affects noticeably the flow structure in the blade cascade. The position and the extent of separation practically do not change with the incidence angle. The flow around the leading edge is sensitive to the incidence angle. However, changes of the flow structure due to the incidence angle can be seen mainly near the leading edge. A better agreement with experimental data can be achieved by the application of the more complex computational domain corresponding with the test section.

\section{Acknowledgements}

The research was supported by the Czech Ministry of Education, Youth and Sports under the project LTAUSA19036 solved in the framework of the Czech-American scientific cooperation in the program INTER-EXCELLENCE and by the project SGS OHK2-004/19. Institutional support RVO 61388998 is also gratefully acknowledged.

\section{References}

[1] Luxa, M., Šimurda, D., Fořt, J., Fürst, J., Šafař́k, P., Synáč, J., Rudas, B.: Aerodynamic Investigation of Tip Section for Titanium Blade 54", Proc of the 11th European Conference on Turbomachinery Fluid Dynamics and Thermodynamics (ETC11), Madrid (2015)

[2] Gaitonde, D. V.: Progress in shock wave/boundary layer interactions, Progress in Aerospace Sciences, 72, 80-99 (2015)

[3] Giepman R.H.M., Schrijer F.F.J., Oudheusden van B. W.: High-resolution PIV measurements of a transitional shock wave-boundary layer interaction, Experiments in Fluids, 56, 113, 1-20 (2015)

[4] Teramoto, S.: Large-eddy simulation of transitional boundary layer with impinging shock wave, AIAA Journal, 43, 2354-2363 (2005)

[5] Piotrowicz M., Flaszyński P.: Numerical investigations of shock wave interaction with laminar boundary layer on compressor profile, IOP Publishing, Journal of Physics: Conference, Series 760, 012023 (2016)

[6] Luxa, M., Příhoda, J., Šimurda, D., Straka, P. Synáč, J.: Investigation of the compressible flow through the tip-section turbine blade cascade with supersonic inlet, Journal of Thermal Science, 25, 1-7 (2016)

[7] Straka, P., Příhoda, J., Fenderl, D., Rudas, B.: Effect of inlet turbulence on compressible flow through the tip-section turbine blade cascade with supersonic inlet, MATEC Web of Conferences, 168, 02007, 9 p. (2018)

[8] Luxa, M., Šimurda D.: Optical and pneumatic measurements on TR-U-8 cascade, Research report Z1558/18, 145 p., Institute of Thermomechanics of the Czech Academy of Sciences, Praha (2018)

[9] Luxa, M., Šimurda D., Hála J.: Measurements on TR-U-9 cascade, Research report Z1610/20, 144 p., Institute of Thermomechanics of the Czech Academy of Sciences, Praha (2020)

[10] Musil, J., Př́ihoda, J., Fürst, J.: Simulation of supersonic flow through the tip-section turbine blade cascade with a flat profile, Proc. Conf. Proceedings of Topical Problems of Fluid Mechanics, Praha, 169-174 (2019) 
[11] Menter, F. R.: Two-equation eddy-viscosity turbulence models for engineering applications, AIAA Journal, 32, 8, 1598-605 (1994)

[12] Langtry, R. B., Menter, F. R.: Correlation-based transition modeling for unstructured parallelized computational fluid dynamics codes, AIAA Journal, 47, 2894-2906 (2009)

[13] Fürst, J.: Development of a coupled matrix-free LU-SGS solver for turbulent compressible flows, Computers \& Fluids, 172, 332-339 (2018)

[14] Louda P., Straka P., Příhoda J.: Simulation of transonic flows through a turbine blade cascade with various prescription of outlet boundary conditions, EPJ Web of Conferences, 180, 02056 (2018) 